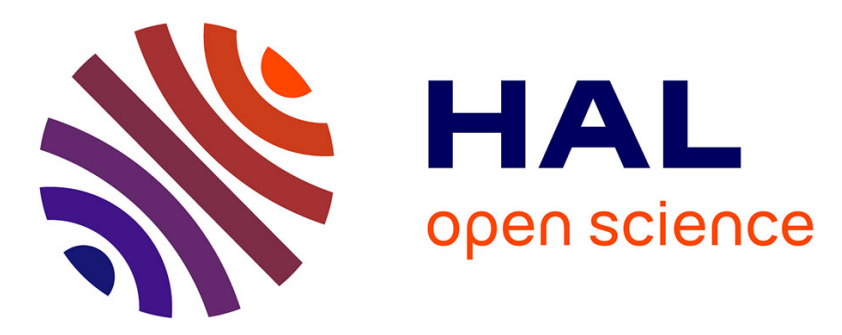

\title{
Vibrational Response of Au-Ag alloy and Porous Au Single nanowires Probed by Ultrafast Pump-Probe Spectroscopy
}

R Delalande, L Burr, E. Charron, M Jouini, M E Toimil-Molares, L Belliard

\section{- To cite this version:}

R Delalande, L Burr, E. Charron, M Jouini, M E Toimil-Molares, et al.. Vibrational Response of AuAg alloy and Porous Au Single nanowires Probed by Ultrafast Pump-Probe Spectroscopy. Applied Physics Letters, 2019, 10.1063/1.5108772 . hal-02134035v2

\section{HAL Id: hal-02134035 \\ https://hal.science/hal-02134035v2}

Submitted on 23 Sep 2020

HAL is a multi-disciplinary open access archive for the deposit and dissemination of scientific research documents, whether they are published or not. The documents may come from teaching and research institutions in France or abroad, or from public or private research centers.
L'archive ouverte pluridisciplinaire HAL, est destinée au dépôt et à la diffusion de documents scientifiques de niveau recherche, publiés ou non, émanant des établissements d'enseignement et de recherche français ou étrangers, des laboratoires publics ou privés. 


\section{Vibrational response of Au-Ag alloy and porous Au single nanowires probed by ultrafast pump-probe spectroscopy}

Cite as: Appl. Phys. Lett. 115, 083103 (2019); https://doi.org/10.1063/1.5108772

Submitted: 02 May 2019. Accepted: 03 August 2019. Published Online: 21 August 2019

R. Delalande (D, L. Burr, E. Charron, M. Jouini, M. E. Toimil-Molares, and L. Belliard

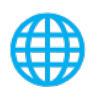

\section{ARTICLES YOU MAY BE INTERESTED IN}

Infrared and optical emission spectroscopy study of atmospheric pressure plasma-enhanced spatial ALD of $\mathrm{Al}_{2} \mathrm{O}_{3}$

Applied Physics Letters 115, 083101 (2019); https://doi.org/10.1063/1.5113753

The effect of luminescent coupling on modulated photocurrent measurements in multijunction solar cells

Applied Physics Letters 115, 083506 (2019); https://doi.org/10.1063/1.5115014

Current-injection quantum-entangled-pair emitter using droplet epitaxial quantum dots on GaAs(111)A

Applied Physics Letters 115, 083106 (2019); https://doi.org/10.1063/1.5103217

\section{Lock-in Amplifiers up to 600 $\mathrm{MHz}$}
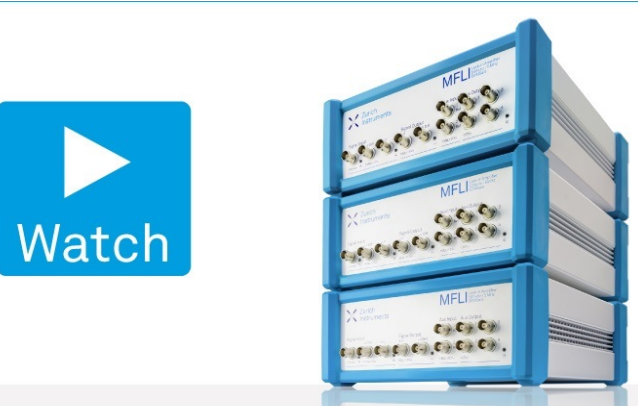


\title{
Vibrational response of Au-Ag alloy and porous Au single nanowires probed by ultrafast pump-probe spectroscopy
}

Cite as: Appl. Phys. Lett. 115, 083103 (2019); doi: 10.1063/1.5108772

Submitted: 2 May 2019 • Accepted: 3 August 2019 .

Published Online: 21 August 2019
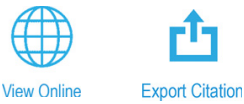

R. Delalande, ${ }^{1}$ (D) L. Burr, ${ }^{2}$ E. Charron, ${ }^{1}$ M. Jouini, ${ }^{7}$ M. E. Toimil-Molares, ${ }^{2}$ and L. Belliard ${ }^{1, a)}$

\author{
AFFILIATIONS \\ ${ }^{7}$ Institut des NanoSciences de Paris, Sorbonne Université, UPMC Universités Paris 06, UMR 7588, Paris F-75005, France \\ ${ }^{2}$ Materials Research Department, GSI Helmhotz Centre for Heavy Ion Research, Planckstr. 1, 64291 Darmstadt, Germany

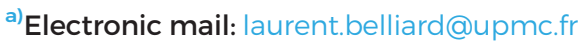

\begin{abstract}
We report on the ultrafast vibrational response of single Au-Ag alloy and Au porous nanowires obtained by the template method. The oscillations of the sample reflectivity are correlated with eigenmodes (EM) confined in the nanowire. Taking advantage of a free standing geometry, we are able to detect few harmonics. Coupling electron microscopy and pump-probe investigations with a very high spectral resolution performed on the same wire, we demonstrate that both longitudinal and transverse sound velocities could be obtained for variable Au concentrations. An additional quadrupolar mode is also observed and reproduced by finite element simulation. Finally, porous nanowires are investigated.
\end{abstract}

Published under license by AIP Publishing. https://doi.org/10.1063/1.5108772

In the past few decades, multicomponent heterostructure nanowires (NWs) have received great interest from the fundamental as well as from the application point of view. This interest originates from the fact that specific functionalities are expected when several materials are purposely combined in the same single nanostructure. ${ }^{1,2}$ Different types of heterostructures such as core-shell, multilayered, alloy, or nanoporous NWs are being developed and characterized and find applications in fields such as magnetism, ${ }^{3-7}$ catalysis, ${ }^{8,9}$ electronics, ${ }^{10-12}$ optics, ${ }^{13-17}$ biological sensing, ${ }^{18}$ fuel cells, ${ }^{19}$ solar energy harvesting, ${ }^{20-22}$ microactuators, ${ }^{23}$ or acoustics. ${ }^{24,25}$

The impact of size reduction down to the nanoscale also attracts considerable attention especially on acoustic responses. Indeed, confinement deeply modifies the acoustic dispersion relations compared with the bulk counterparts. This, in turn, strongly influences the thermal ${ }^{26,27}$ and electronic ${ }^{28}$ properties of the nanostructures, characteristics which must be carefully taken into consideration during the development of NW based thermoelectric, photonic, or electronic devices. ${ }^{27}$

The Young's modulus of nanostructures has been determined using various techniques, such as atomic force microscopy, ${ }^{32}$ transmission electron microscopy, ${ }^{33}$ or, alternatively, time-resolved pump and probe spectroscopy where an ultrashort femtosecond pump pulse induces a fast heating. ${ }^{34}$ The excited eigenmode (EM) of the nanoobject is subsequently optically detected by a time-delayed probe pulse.
This pump-probe method is nowadays considered a very powerful tool to address local elasticity issues into nanostructures, particularly interesting for being contact-less and for not requiring special sample preparation.

Since the pioneering work of van Dijk, ${ }^{35}$ who investigated the dynamic response of single gold nanospheres, a large number of materials and nano-object shapes have been investigated. ${ }^{36-42}$ There have been a number of publications on semiconductor ${ }^{43-45}$ and single component metal nanowires. ${ }^{46-51}$

In this letter, we report on the dynamic response of single Au-Ag alloy NW. As we show, the vibration mode may be tuned by changing the composition, just like its surface plasmon resonance, ${ }^{52}$ and therefore, the guided acoustic properties may be controlled. Single Au-Ag alloy NWs with a nominal diameter of $200 \mathrm{~nm}$, a length of few $\mu \mathrm{m}$, and controlled gold molar fractions of 30,40 , and $60 \%$ have been considered. In addition, Ag is selectively dealloyed from the NWs, and the properties of the resulting porous Au NWs are also examined. Due to their extremely large surface, nanoporous NWs are very attractive for different applications in, e.g., optics, ${ }^{53}$ catalysis, ${ }^{54}$ corrosion, ${ }^{55}$ and chemical $^{56}$ and biological sensing. ${ }^{57}$ Their dynamic response is expected to be correlated with the thermal conductivity decrease, which represents a promising track toward the increase in thermoelectric efficiency. ${ }^{58}$ In order to improve the measurements, individual 
NWs are transferred onto prestructured substrates with $\mu$ m-wide trenches. Indeed, when a NW rests free-standing on a cavity or trench, the energy relaxation channel through the substrate is suppressed and the NW nanoresonators exhibit higher quality factors. ${ }^{48}$ Applying this geometry, breathing harmonics and quadrupole mode response have been observed. The standard Pochhammer-Chree model coupled with scanning electron microscopy (SEM) measurements is combined to successfully obtain a complete characterization of the longitudinal and transverse sound velocities in the Au-Ag NWs for each Au concentration. Finite element simulations are also performed in order to complete our investigation and interpretations.

The NWs were synthesized by electrodeposition in etched iontrack membranes. First, $30 \mu$ m thick polycarbonate foils (Makrofol N, Bayer AG) were irradiated with $2 \mathrm{GeV}$ Au ions at the UNILAC linear accelerator of GSI Helmholtz Center (Darmstadt, Germany). Prior to etching, each side of the foils is exposed to UV light for $1 \mathrm{~h}$. The iontracks created by ions are subsequently selectively dissolved in a $2 \mathrm{M}$ $\mathrm{NaOH}$ solution at $50^{\circ} \mathrm{C}$ for $30 \mathrm{~min}$, resulting in cylindrical nanochannels with an average diameter of $200 \mathrm{~nm}$. After etching, a thick $\mathrm{Au}$ layer is sputtered on one side of the polymer membrane and reinforced with an electrodeposited Au layer (thickness of few $\mu \mathrm{m}$ ). This layer acts as a working electrode during the deposition of $\mathrm{Au}$ and $\mathrm{Au}-$ $\mathrm{Ag}$ inside the nanochannels. The details of the electrodeposition of $\mathrm{Au}$ and $\mathrm{Au}-\mathrm{Ag}$ alloy NWs have been previously published. ${ }^{55}$ The polymer membrane is then removed by immersing the sample in several baths of dichloromethane solution, and the NWs are detached from the Au electrode by a short sonication on clean dichloromethane. Finally, individual Au-Ag NWs are pipetted onto the prestructured Si substrates. The process is optimized such that enough NWs are freestanding between two adjacent beams. To produce porous Au wires, the substrate with the Au-Ag NWs is immersed in a nitric acid solution for $3 \mathrm{~h}$, rinsed with 3 subsequent MilliQ water baths, and dried in air. Thanks to transmission electron microscopy as well as energydispersive X-ray spectroscopy, the homogeneity of the body of NWs has been highlighted for Au-Ag alloys. ${ }^{55}$ Nevertheless, a surface enrichment of few $\mathrm{nm}$ of the main metal has also been pointed out, meaning that a Ag-rich layer in $\mathrm{Au}_{0.4} \mathrm{Ag}_{0.6} \mathrm{NWs}$ can be observed for example.

The experimental setup used during this study working in reflection geometry in an inverse Olympus microscope has been described in detail in previous works. ${ }^{59,60}$ Ultrafast pump-probe spectroscopy experiments are performed using a mode-locked Ti:sapphire (MAI-TAI Spectra) laser source operating at $800 \mathrm{~nm}$ with a pulse duration below $100 \mathrm{fs}$ at the laser output and a repetition rate of $78.8 \mathrm{MHz}$. Synchronous detection on the sample reflectivity is performed by modulating the pump beam at $1.8 \mathrm{MHz}$. Both pump and probe beams are focused by means of a microscope objective with $\mathrm{NA}=0.9$ and are normally incident on the sample. The laser spots can be focused around a $1 \mu \mathrm{m}$ diameter at $1 / \mathrm{e}^{2}$. A two-color experiment is performed by doubling the pump frequency $(400 \mathrm{~nm})$ with a nonlinear crystal (Beta barium borate or BB0) to avoid scattered light coming from the pump beam. A dichroic filter located in front of the diode system suppresses the light of the pump beam, its power on the sample is reduced around $300 \mu \mathrm{W}$, and the power of the probe beam does not exceed $50 \mu \mathrm{W}$.

Figure 1 presents the oscillations of reflectivity $\Delta R / R$ measured on a $200 \mathrm{~nm}$ diameter single Au NW above a trench. A SEM image of

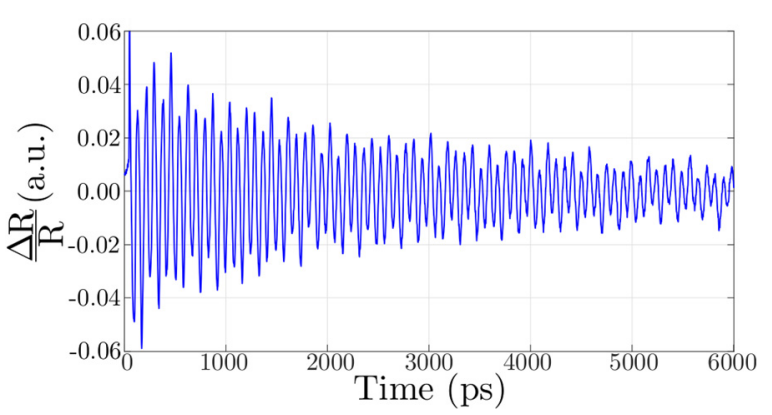

FIG. 1. Time resolved reflectivity response measured on a single $200 \mathrm{~nm}$ diameter Au nanowire placed across a trench.

this NW is shown in Fig. 5(b). The subsequent frequency analysis of the reflectivity signal is shown in Fig. 2. It evidences a main frequency component around $f_{2}=12.2 \mathrm{GHz}$ as well as several other components at $f_{3}=29.3$ and $f_{4}=46.3 \mathrm{GHz}$ and a low-frequency component around $f_{1}=5.0 \mathrm{GHz}$. This obtained spectrum remains unchanged along the wire.

Those components reveal the vibrations of the Au NW. In particular, the components numbered as $f_{2}, f_{3}$, and $f_{4}$ correspond to the first three breathing modes (BMs) of the wire exhibiting pure radial displacement. This is found by solving the Navier equation in an infinite isotropic NW in order to obtain the Pochhammer-Chree equation ${ }^{61,62}$ which can be approximated for such a mode in the long wavelength assumption under the form

$$
2 \frac{v_{T}}{v_{L}} J_{1}\left(\frac{\omega_{n} a}{v_{L}}\right)-\frac{\omega_{n} a}{v_{T}} J_{0}\left(\frac{\omega_{n} a}{v_{L}}\right)=0,
$$

where $a$ is the wire radius, $J_{0}$ and $J_{1}$ correspond to the Bessel function of the zeroth and first kind, respectively, $\omega_{n}=2 \pi f_{n}$ is the pulsation of mode $n$, and $v_{L}$ and $v_{T}$ are the longitudinal and transverse sound velocities. Of course, experimental NWs are not infinitely long but this assumption can be done as their length is much larger than the pump and probe beams. Surface effects and nonlocality phenomena are neglected due to the scale of the understudy of the NWs.

However, even if the frequencies of these 3 modes are known, the three unknown parameters $a, v_{L}$, and $v_{T}$ cannot yet be unambiguously

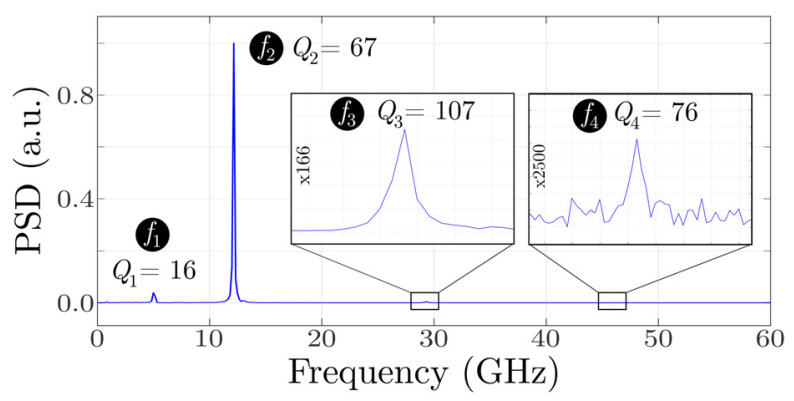

FIG. 2. Normalized power spectral density of the signal shown in Fig. 1 presenting 3 frequency components at $f_{2}=12.1, f_{3}=29.3$, and $f_{4}=46.3 \mathrm{GHz}$ assigned to radial breathing modes as well as a lower signature at $f_{1}=5.0 \mathrm{GHz}$ related to a quadrupolar mode geometry. 
determined due to the mathematical structure of Eq. (1). This is circumvented by determining the radius of the excited NW, $a$, by SEM. For this, after the pump-probe measurements, the same NW was located under the SEM beam using the numbered marks on the trenches.

Subsequently, the $\left(v_{L} v_{T}\right)$ couple solutions of Eq. (1) were determined for each frequency for the measured value of $a$. Figure 3 shows the transverse velocities, plotted as a function of the longitudinal ones. Three different curved lines are obtained. As these three frequency components are simultaneously observed on the spectrum, the unique possible $\left(v_{L}, v_{T}\right)$ couple is obtained by the intersection point.

The slope of these curves increases with the harmonic order, indicating that the resonance frequency is less and less sensitive to the transverse velocity as it has been previously demonstrated. ${ }^{34}$ As shown in Fig. 3, a single intersection point is not obtained, but instead there are three intersection points quite close to each other. It leads to the average values $v_{L}^{M}=3378 \mathrm{~m} \cdot \mathrm{s}^{-1}$ and $v_{T}^{M}=1381 \mathrm{~m} \cdot \mathrm{s}^{-1}$ as well as the standard deviations $\sigma_{v_{L}}=4 \mathrm{~m} \cdot \mathrm{s}^{-1}$ and $\sigma_{v_{T}}=86 \mathrm{~m} \cdot \mathrm{s}^{-1}$. We would like to emphasize that the relative standard deviations obtained are $0.12 \%$ and $6.2 \%$ for the longitudinal and transverse velocities, respectively.

To investigate how velocities vary as a function of the NW composition, several NWs with the same nominal diameter but different molar fractions of gold and silver (namely, $\mathrm{Au}_{0.3} \mathrm{Ag}_{0.7}, \mathrm{Au}_{0.4} \mathrm{Ag}_{0.6}$, and $\mathrm{Au}_{0.6} \mathrm{Ag}_{0.4}$ ) were characterized in the same manner as described above. However, in the case of alloy wires, the analysis was based on only 2 frequency components, since only two BMs were recorded in most of the cases. As the molar fraction of gold decreases, the frequencies of the breathing mode slightly increase, which is the tendency one can expect looking at the elastic constants of bulk pure silver and gold, respectively. Simultaneously, two homogenization models are used to calculate both velocities from the Young's modulus, Poisson's ratio, and density of bulk gold and silver properties given in Table I. The obtained results are plotted in Fig. 4(a) together with the experimental values.

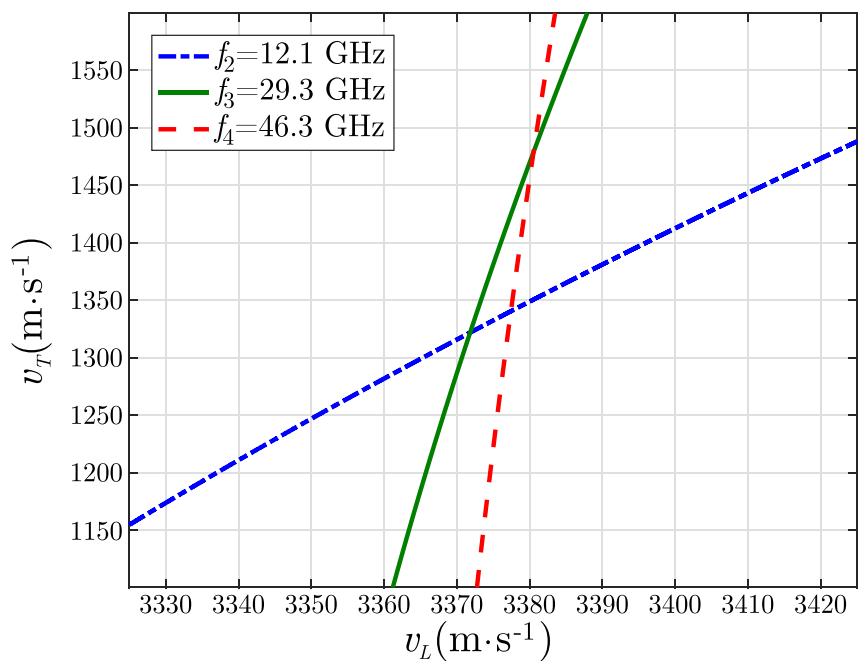

FIG. 3. Couples of longitudinal and transverse velocity solutions of Eq. (1) for the first three breathing mode frequencies for $2 a=200.1 \mathrm{~nm}$.
TABLE I. Values of the Young's Modulus E, Poisson's ratios $\gamma$, and the densities $\rho$ of gold and silver.

\begin{tabular}{lccc}
\hline \hline Materials & $E(\mathrm{GPa})$ & $\gamma$ & $\rho\left(\mathrm{kg} \mathrm{m}^{-3}\right)$ \\
\hline $\mathrm{Au}$ & 78 & 0.43 & $1.93 \times 10^{4}$ \\
$\mathrm{Ag}$ & 84 & 0.37 & $1.05 \times 10^{4}$ \\
\hline \hline
\end{tabular}

As shown in Fig. 4, both transverse and longitudinal velocities obtained experimentally increase as the gold molar fraction decreases. This tendency is coherent with the prediction made from the Voigt and Reuss models. Nevertheless, one can notice that the relative dispersion of the experimental values from the models is more important for the transverse than for the longitudinal velocity. This is a consequence of the BM being less sensitive to the former than to the latter. A more important scattering of the experimental values for a lower gold molar fraction is distinguishable for both velocities. SEM investigations reveal an increase in surface roughness or the presence of particles on the surface of NWs with a higher Ag concentration [Fig. 5(a)], whereas the Au NWs are smooth [Fig. 5(b)]. Coupled to the surface enrichment, this could cause a change of the BM frequencies that could explain the scattering of values observed for a higher silver molar fraction. It is also shown in Fig. 4(b) that for higher Ag concentrations, quality factors are smaller for both BMs.

The first component of the spectrum shown in Fig. 2, i.e., $f_{1}$, can also be used in order to determine the mechanical properties of the NW. The velocities obtained experimentally by the BM method lead, with the density of bulk $\mathrm{Au}$, to the experimental values for both Young's modulus $E_{\text {exp }}=90.8 \pm 8.1 \mathrm{GPa}$ and Poisson's ratio $\nu_{\exp }$ $=0.41 \pm 0.01$ which happened to be slightly different from the Au bulk. These mechanical parameters can then be used to run finite element simulations using the COMSOL software to estimate nonpure isotropic radial EM, and the details of the calculations can be found in

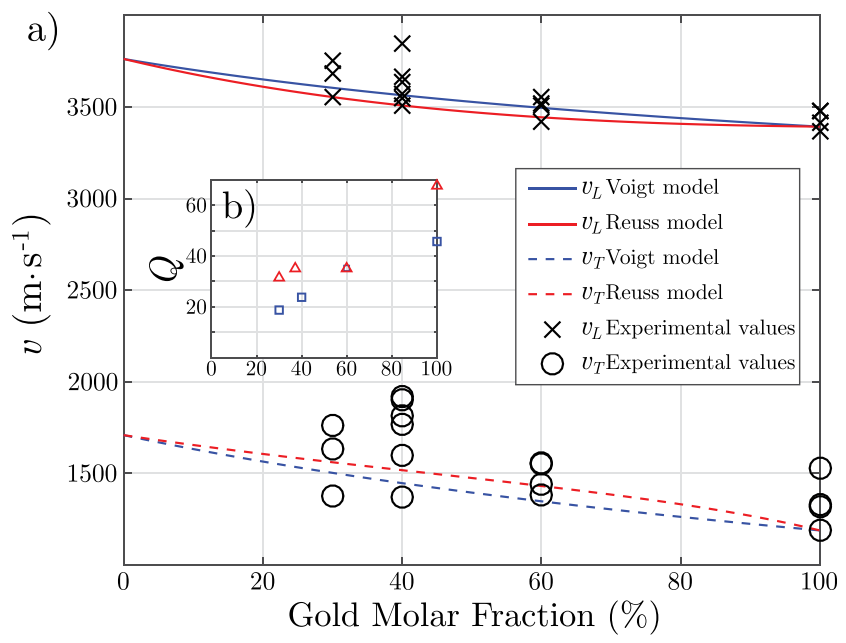

FIG. 4. (a) Transverse and longitudinal velocities obtained experimentally compared to those obtained thanks to Voigt and Reuss models. (b) Average quality factors obtained experimentally for each composition for the first (blue squares) and the second (red triangles) breathing modes. 

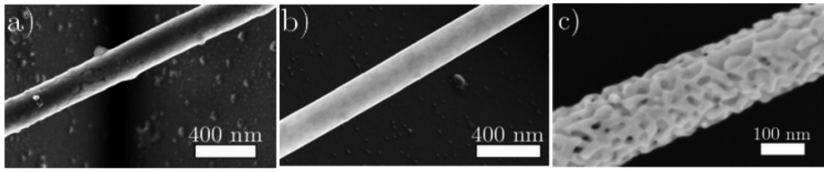

FIG. 5. Scanning electron microscopy images of (a) $\mathrm{Au}_{0.3} \mathrm{Ag}_{0.7}$, (b) $\mathrm{Au}_{1.0}$, and (c) porous Au with $\phi=0.6$ nanowires.

Ref. 63. Among such modes, a quadrupolar mode can be identified at $4.94 \mathrm{GHz}$, very close to the experimental value of $f_{1}$ shown in Fig. 2. Figure 6(b) represents its mode shape. A relative error on the frequency less than $4 \%$ may be deduced from measurements performed on multiple NWs, proof that the estimations of both velocities are coherent. Photonic EM exhibiting such angular symmetry has been recently reported in $\mathrm{Si} \mathrm{NW}^{64}$ and may be assigned to the quadrupolar phonon mode excitation. Naturally, the efficiency of such a mechanism is much lower than the uniform heat excitation which is at the origin of the $\mathrm{BM}$ generation.

The sensitivity of this quadrupolar mode to both longitudinal and transverse velocities can be investigated by running finite element simulations for different velocity couples and extracting the frequency of the mode. The obtained results for different values of $v_{L}$ while $v_{T}$ is fixed, and vice versa, represented in Fig. 6 indicate that this mode is more sensitive to the transverse than to the longitudinal velocity.

It is proposed to use the value of $f_{1}$ measured experimentally as well as the value of $v_{L}$ obtained by the BM method in order to determine a more accurate value of $v_{T}$. Using this method and some finite element simulations, one can improve the estimation of $v_{T}$ from $1381 \pm 86 \mathrm{~m} \cdot \mathrm{s}^{-1}$ obtained from Fig. 3 to $1336 \pm 22 \mathrm{~m} \cdot \mathrm{s}^{-1}$.

As shown in Fig. 6, when the fixed value of $v_{L}$ used is the one given by the BM method and the fixed value of $v_{T}$ is the one obtained by this second method, the intersection point of those two curves has

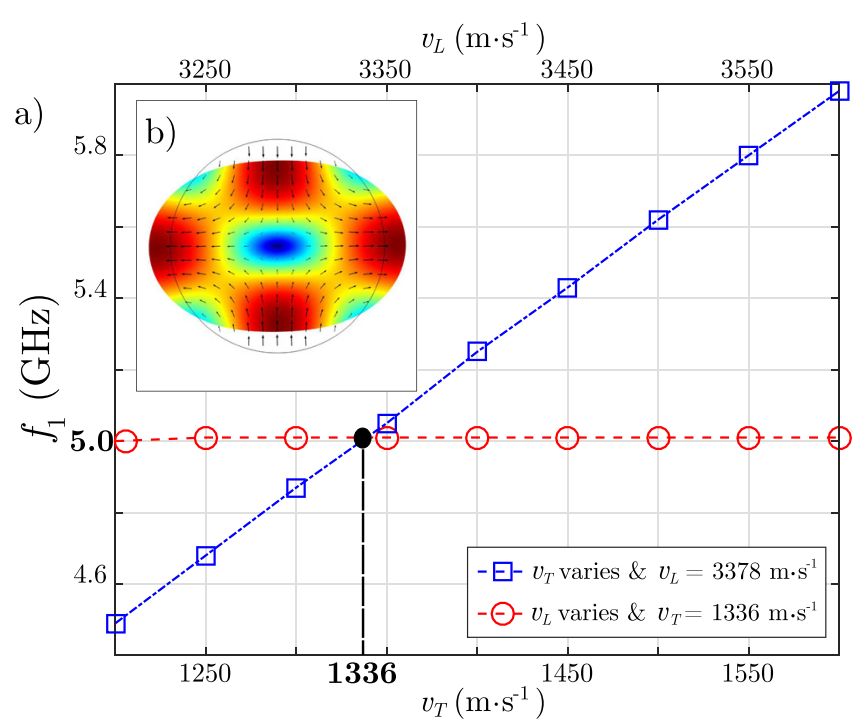

FIG. 6. (a) Variation of $f_{1}$ for a given value of $v_{L}$ and varying $v_{T}$ (blue) and for a given value of $v_{T}$ and varying $v_{L}$ (red). (b) Mode shape calculated by finite element simulation. for ordinate the value of $f_{1}$ obtained experimentally. Knowing $f_{1}$ and $v_{L}$, the accurate value of $v_{T}$ can then be obtained graphically. This added process allows one to obtain a more accurate value of the transverse velocity in addition to the accurate value for the longitudinal velocity from the BM method. Nevertheless, it requires us to measure the frequency of the quadrupolar mode.

Porous NWs obtained after dealloying were also investigated. Porosity $\phi$ is defined as the Ag volumetric fraction before this process. $^{17}$ Time resolved reflectivity responses and the corresponding power spectral densities obtained on $\mathrm{Au}_{0.4} \mathrm{Ag}_{0.6} \mathrm{NWs}$ before and after dealloying are shown in Figs. 7(a) and 7(b), respectively. While the oscillations last for several ns for the alloy NWs, they are mostly shorter than $1 \mathrm{~ns}$ in the case of the porous ones. This results in a much smaller quality factor for the first breathing mode, as it is shown in Fig. 7(b), which moreover happens to be the only detected component. As shown in Fig. 7(d), an increase in the damping characterized by a decrease in the quality factor as the porosity increases may be associated with the number of diffusion barriers which increases with the porosity level. The results are in good agreement with very low thermal conductivity reported in porous NWs. ${ }^{58}$ Nevertheless, a clear shift to lower frequencies after dealloying is observed in Figs. 7(b) and 7(c), signature of a large decrease in the stiffness in such granular medium.

We have shown that pump-probe investigations with a very high spectral resolution can be performed to detect BMs of a single NW, made of, in our case, Au-Ag alloys with different compositions. Thanks to a prestructured substrate suppressing its mechanical coupling with the NWs, several orders of EMs up to more than $46 \mathrm{GHz}$ can be distinguished with high quality factors. Solving the Pochhammer-Chree equation, both longitudinal and transverse velocities can be determined, the latter being rarely addressed in the literature. The detection of an additional mode at lower frequency allows us to increase the accuracy of the transverse velocity determination using the finite element approach. This allowed us to verify Voigt and Reuss a)
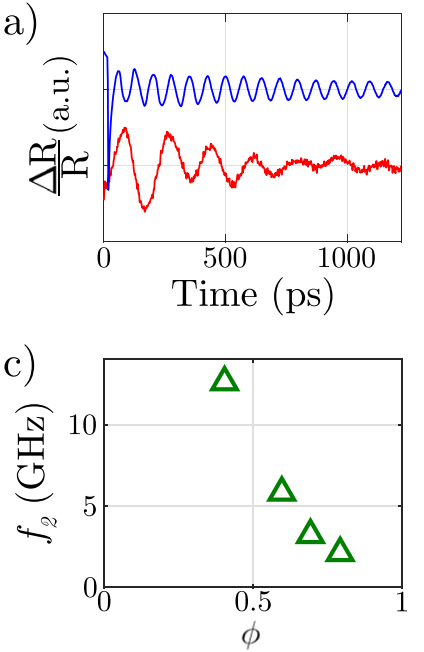

b)

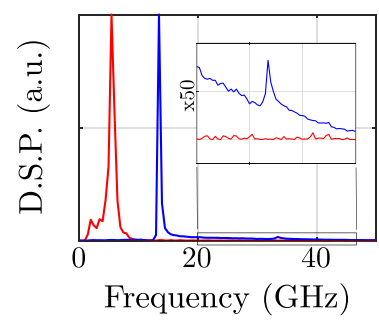

FIG. 7. (a) Time resolved reflectivity responses measured on a single $\mathrm{Au}_{0.4} \mathrm{Ag}_{0.6}$ alloy nanowire (blue) and a Au porous nanowire with a porosity of $\phi=0.6$ (red). (b) Corresponding power spectral densities showing $f_{2}=13.5 \mathrm{GHz}$ and $Q_{2}=24$ for the alloy and $f_{2}=5.5 \mathrm{GHz}$ and $Q_{2}=6$ for the porous one. (c) and (d) Frequency and quality factor of the first breathing mode as a function of the porosity. 
models for Au-Ag NWs. Similar investigation has been realized on porous $\mathrm{Au}$ NWs. We have demonstrated that ultrafast pump-probe spectroscopy can be used in order to evaluate the elastic properties of single NW and could be used for porous NWs.

The employed NWs are part of the experiment UMAT, which was performed at the beam line X0 at the GSI Helmhotzzentrum fuer Schwerionenforschung, Darmstadt (Germany), in the frame of FAIR Phase-0.

\section{REFERENCES}

${ }^{1}$ L. Piraux, J. M. George, J. F. Despres, C. Leroy, E. Ferain, R. Legras, K. Ounadjela, and A. Fert, Appl. Phys. Lett. 65, 2484 (1994).

${ }^{2}$ J. J. Mock, S. J. Oldenburg, D. R. Smith, D. A. Schultz, and S. Schultz, Nano Lett. 2, 465 (2002).

${ }^{3}$ D. Zhang, Z. Liu, S. Han, C. Li, B. Lei, M. P. Stewart, J. Tour, and C. Zhou, Nano Lett. 4, 2151 (2004).

${ }^{4}$ L. Piraux, S. Duboix, A. Fert, and L. Belliard, Eur. Phys. J. B 4, 413 (1998).

${ }^{5}$ Y. T. Chong, D. Görlitz, S. Martens, M. Y. E. Yau, S. Allende, J. Bachmann, and K. Nielsch, Adv. Mater. 22, 2435 (2010).

${ }^{6}$ K. Kiani, J. Phys. Chem. Solids 95,89 (2016).

${ }^{7}$ K. Kiani, Mater. Chem. Phys. 162, 531 (2015).

${ }^{8}$ Y. Ding, M. Chen, and J. Erlebacher, J. Am. Chem. Soc. 126, 6876 (2004).

${ }^{9}$ X. Zhang and Y. Ding, Catal. Sci. Technol. 3, 2862 (2013).

${ }^{10}$ E. D. Herderick, J. S. Tresback, A. L. Vasiliev, and N. P. Padture, Nanotechonology 18, 155204 (2007).

${ }^{11}$ L. J. Lauhon, M. S. Gudiksen, D. Wang, and C. M. Lieber, Nature 420, 57 (2002).

${ }^{12}$ M. Cassinelli, S. Müller, K. O. Voss, C. Trautmann, F. Völkein, J. Gooth, K. Nielsch, and M. E. Toimil-Molares, Nanoscale 9, 3169 (2017).

${ }^{13}$ J. Zhu, Nanoscale Res. Lett. 4, 977 (2009).

${ }^{14}$ J. Goldberger, R. He, Y. Zhang, S. Lee, H. Yan, H. J. Choi, and P. Yang, Nature 422, 599 (2003).

${ }^{15}$ C. D. Keating and M. J. Natan, Adv. Mater. 15, 451 (2003).

${ }^{16}$ S. R. Nicewarner-Peña, A. J. Carado, K. E. Shale, and C. D. Keating, J. Phys. Chem. B. 107, 7360 (2003).

${ }^{17}$ I. Schubert, C. Huck, P. Kröber, F. Neubrech, A. Pucci, M. E. Toimil-Molares, C. Trautmann, and J. Vogt, Adv. Opt. Mater. 4, 1838 (2016).

${ }^{18}$ S. O. Kucheyev, J. R. Hayes, J. Biener, T. Huser, C. E. Talley, and A. V. Hamza, Appl. Phys. Lett. 89, 053102 (2006).

${ }^{19}$ R. Zeis, A. Mathur, G. Fritz, J. Lee, and J. Erlebacher, J. Power Sources 165, 65 (2007).

${ }^{20}$ M. Law, L. E. Greene, A. Radenovic, T. Kuykendall, J. Liphardt, and P. Yang, J. Phys. Chem. B. 110, 22652 (2006).

${ }^{21}$ L. E. Greene, M. Law, B. D. Yuhas, and P. Yang, J. Phys. Chem. C. 111, 18451 (2007).

${ }^{22}$ J. A. Czaban, D. A. Thompson, and R. R. LaPierre, Nano Lett. 9, 148 (2009).

${ }^{23}$ D. Kramer, R. N. Viswanath, and J. Weissmüller, Nano Lett. 4, 793 (2004).

${ }^{24}$ C. Jean, L. Belliard, T. W. Cornelius, O. Thomas, Y. Pennec, M. Cassinelli, M. E. Toimil-Molares, and B. Perrin, Nano Lett. 16, 6592 (2016).

${ }^{25}$ F. Xu, Y. Guillet, S. Ravaine, and B. Audoin, Phys. Rev. B 97, 165412 (2018).

${ }^{26}$ N. Mingo, L. Yang, D. Li, and A. Majumdar, Nano Lett. 3, 1713 (2003).

${ }^{27}$ A. Boukai, Y. Bunimovich, J. Tahir-Kheli, J.-K. Yu, W. A. Goddard III, and J. R. Heath, Nature 451, 168 (2008).

${ }^{28}$ N. Bannov, V. Aristov, V. Mitin, and M. A. Stroscio, Phys. Rev. B 51, 9930 (1995).
${ }^{29}$ D. Li, Y. Wu, P. Kim, L. Shi, P. Yang, and A. Majumdar, Appl. Phys. Lett. 83, 2934 (2003).

${ }^{30}$ A. I. Hochbaum, R. Chen, R. D. Delgado, W. Liang, E. C. Garnett, M. Najarian, A. Majumdar, and P. Yang, Nature 451, 163 (2008).

${ }^{31}$ J. Krieg, R. Giraud, H. Funke, J. Dufouleur, W. Escoffier, C. Trautmann, and M. E. Toimil-Molares, J. Phys. Chem. Solid 128, 360 (2019).

${ }^{32}$ E. W. Wong, P. E. Sheehan, and C. M. Lieber, Science 277, 1971 (1997).

${ }^{33}$ C. Y. Nam, P. Jaroenapibal, D. Tham, D. E. Luzzi, S. Evoy, and J. E. Fischer, Nano Lett. 6, 153 (2006).

${ }^{34}$ A. Crut, P. Maioli, N. Del Fatti, and F. Vallée, Chem. Soc. Rev. 43, 3921 (2014).

${ }^{35}$ M. A. van Dijk, M. Lippitz, and M. Orrit, Phys. Rev. Lett. 95, 267406 (2005).

${ }^{36}$ J. Burgin, P. Langot, N. Del Fatti, F. Vallée, W. Huang, and M. A. El-Sayed, J. Phys. Chem. C 112, 11231 (2008).

${ }^{37}$ R. Marty, A. Arbouet, C. Girard, A. Mlayah, V. Paillard, V. K. Lin, S. L. Teo, and S. Tripathy, Nano Lett. 11, 3301 (2011).

${ }^{38}$ T. A. Kelf, Y. Tanaka, O. Matsuda, E. M. Larsson, D. S. Sutherland, and O. B. Wright, Nano Lett. 11, 3893 (2011).

${ }^{39}$ H. Staleva and G. V. Hartland, J. Phys. Chem. C 112, 7535 (2008).

${ }^{40}$ P. Zjilstra, A. L. Tchebotareva, J. W. Chon, M. Gu, and M. Orrit, Nano Lett. 8, 3493 (2008).

${ }^{41}$ P. M. Jais, D. B. Murray, R. Merlin, and A. V. Bragas, Nano Lett. 11, 3685 (2011).

${ }^{42}$ V. Juvé, A. Crut, P. Maioli, M. Pellarin, M. Broyer, N. Del Fatti, and F. Vallée, Nano Lett. 10, 1853 (2010).

${ }^{43}$ S. C. Yang, Y. C. Wu, P. A. Mante, C. C. Chen, H. P. Chen, H. Y. Chou, M. H. Shih, and C. K. Sun, Appl. Phys. Lett. 105, 243101 (2014).

${ }^{44}$ P. A. Mante, C. Y. Ho, L. W. Tu, and C. K. Sun, Opt. Exp. 20, 18717 (2012).

${ }^{45}$ P. A. Mante, Y. C. Wu, C. Y. Ho, and C. K. Sun, Nano Lett. 13, 1139 (2013).

${ }^{46}$ H. Staleva and G. V. Hartland, Adv. Funct. Mater. 18, 3809 (2008).

${ }^{47}$ T. A. Major, A. Crut, B. Gao, S. S. Lo, N. Del Fatti, F. Vallée, and G. V. Hartland, Phys. Chem. Chem. Phys. 15, 4169 (2013).

${ }^{48}$ L. Belliard, T. W. Cornelius, B. Perrin, N. Kacemi, L. Beccera, O. Thomas, M. E. Toimil-Molares, and M. J. Cassinelli, J. Appl. Phys. 114, 193509 (2013).

${ }^{49}$ T. Devkota, D. Chakraborty, K. Yu, G. Beane, J. E. Sader, and G. V. Hartland, Phys. Chem. Chem. Phys. 20, 17687 (2018).

${ }^{50}$ A. G. N. Sofiah, M. Samykano, K. Kadirgama, R. V. Mohan, and N. A. C. Lah, Appl. Mater. Today 11, 320 (2018).

${ }^{51}$ D. K. Kehoe, S. A. McCarthy, and Y. K. Gun'ko, Nanoscale 11, 4328 (2019).

${ }^{52}$ R. Ferrando, J. Jellinek, and R. L. Johnston, Chem. Rev. 108, 845 (2008).

${ }^{53}$ J. C. Costa, P. Corlo, and P. H. Camargo, RSC Adv. 2, 9801 (2012).

${ }^{54}$ M. Mohl, A. Kumar, A. L. M. Reddy, A. Kukovecz, Z. Konya, I. Kiricsi, R. Vajtai, and P. M. Ajayan, J. Phys. Chem. C 114, 389 (2010).

${ }^{55}$ L. Burr, I. Schubert, W. Sigle, C. Trautmann, and M. E. Toimil-Molares, J. Phys. Chem. C. 119, 20949-20956 (2015).

${ }^{56}$ Z. Liu and P. C. Searson, J. Phys. Chem. B 110, 4318 (2006).

${ }^{57} \mathrm{X}$. Zhang, D. Li, L. Bourgeois, H. Wang, and P. A. Webley, ChemPhysChem 10, 436 (2009).

${ }^{58}$ T. Zhang, S. Wu, J. Xu, R. Zheng, and G. Cheng, Nano Energy 13, 433 (2015).

${ }^{59}$ T. Bienvillle, J. F. Robillard, L. Belliard, I. Roch-Jeune, A. Devos, and B. Perrin, Ultrasonics 44, e1289 (2006).

${ }^{60}$ C. Jean, L. Belliard, L. Becerra, and B. Perrin, Appl. Phys. Lett. 107, 193103 (2015).

${ }^{61}$ L. Pochhammer and J. Reine, Angew. Math. 81, 324 (1876).

${ }^{62}$ C. Chree, Trans. Cambridge Philos. Soc. 14, 250 (1889).

${ }^{63}$ A. Amziane, L. Belliard, F. Decremps, and B. Perrin, Phys. Rev. B 83, 014102 (2011).

${ }^{64}$ P. A. Mante, L. Belliard, and B. Perrin, Nanophotonics 7, 1759 (2018). 